\title{
Crystallography beyond the Bragg Peaks
}

\author{
Thomas Weber*
}

\begin{abstract}
Diffuse scattering contains information on the real structure of disordered crystals that cannot be deduced from conventional structure analysis based on Bragg reflections. In this article the historical development, current state-of-the-art and future perspectives of diffuse scattering are described. The focus is on the last twenty years, in which progress in X-ray instrumentation and computer-aided modeling has transformed diffuse scattering research from a qualitative to a quantitative discipline. Examples of cutting edge diffuse scattering experiments and modern real structure modeling techniques are given.
\end{abstract}

Keywords: Diffuse scattering $\cdot$ Disorder $\cdot$ Modeling $\cdot 3 D-\Delta$ PDF $\cdot$ X-ray detectors

\section{Introduction}

There is no doubt that the greatest success of crystallography is the more or less automatic determination of crystal structures using diffraction methods. Structure investigations, which represented a full $\mathrm{PhD}$ thesis only a few decades ago, may nowadays be accomplished within a day. Modern diffractometers and computer programs make Bragg data collection, structure solution, least-squares refinement and validation of crystal structures almost as simple as pressing a button. However, structure models based only on Bragg reflections are incomplete, because focusing on sharp diffraction patterns implies space and time averaging over all unit cells. The averaging transforms disordered crystals lacking perfect three-dimensional (3D) periodicity into 'ordered' $3 \mathrm{D}$ periodic structures, which show non-physical features such as atoms with fractional occupancy, with multiple positions in the unit cell and with unreasonably oblate and prolate ellip-

${ }^{*}$ Correspondence: Dr. T. Weber ETH Zürich

Laboratorium für Kristallographie

Vladimir-Prelog-Weg 5

$\mathrm{CH}-8093$ Zürich

Tel.: +41446326404

E-mail: thomas.weber@mat.ethz.ch soids. The more disordered a crystal structure is the less meaningful is the information that can be extracted from the average unit cell, e.g. the interatomic distances or the local arrangement of chemical building blocks. The missing information may be recovered from the diffuse part of the diffraction pattern. Although diffuse scattering could in principle be measured as a side product of Bragg data collection, it has frequently been ignored in the past, because the powerful tools necessary to measure and model diffuse scattering were not available. In recent years the demand for studying as a matter of routine the almost white map of disordered crystal structures and their chemical or physical properties has increased, especially in the material sciences. This paper will give a short survey about historical developments, the current state-of-the-art in and future possibilities of diffuse scattering research.

\section{Advances in Diffuse Scattering Experiments}

Since the early days of X-ray diffraction 100 years ago until the 1980 s the typical setup of diffuse scattering experiments hardly changed. X-ray tubes or rotating anodes were the standard radiation sources and the signals were recorded with photographic films. Rotating crystal, precession and Weissenberg techniques yielded twodimensional (2D) projections or sections of reciprocal space once the crystal had been carefully oriented. Exposure times for obtaining 2D diffuse scattering patterns were often on the order of days and measurements of complete 3D diffuse scattering were not feasible at all. Due to the lack of complete and quantitative data, results of disorder studies were semi-quantitative at best.

In the 1990s the situation changed completely. The first powerful area detectors such as image plates and charge-coupled device (CCD) cameras were commercialized and many synchrotron facilities were built. The new experimental possibilities allowed measurements of complete and quantitative three-dimensional data sets within hours. Furthermore, software was developed that permitted reconstructions of arbitrary two- or three-dimensional portions of reciprocal space from the diffraction patterns of randomly oriented crystals. ${ }^{[1]}$ Despite this significant technical progress the performance of CCDs and image plates is still limited and represents a severe bottleneck in diffuse scattering studies. Diffuse scattering experiments with CCD detectors are difficult, because the significant intrinsic detector noise may easily disguise weak and broad diffuse signals. Furthermore, CCDs tend to affect diffuse scattering measurements by artifacts such as so-called streaking or bleeding next to overexposed Bragg reflections. Image plates are less sensitive to artifacts due to saturation and the detector noise is much weaker compared to the one of CCDs, but image plates are slow. While read-out times of CCDs are in the order of a few seconds, reading and erasing a single image plate frame takes more than a minute. The long read-out times of image plates reduce the performance of image plate experiments significantly, especially for synchrotron measurements, where exposure times are only a few seconds per frame.

The next big step in X-ray diffraction instrumentation was the development of pixel detectors such as the Pilatus detector a few years ago. ${ }^{[2]}$ Pixel detectors are ideal for diffuse scattering measurements, because they are very fast, they have a larger dynamic range than CCDs or image plates, they show no intrinsic noise and they allow suppression of fluorescence scattering. Since each pixel has its own read-out electronics, the detector resolution function is essentially one pixel broad. Even strongly 
overexposed pixels do not affect neighboring pixels. This feature makes pixel detectors the instrument of choice for measuring both weak and strong signals even if they are close to each other. Currently, pixel detectors are still expensive and mainly found at synchrotron beam lines, but with manufacturers now offering smaller and cheaper units the number of in-house installations is increasing.

The combination of synchrotron radiation with modern pixel detectors has opened new opportunities for real structure studies that were far from being feasible only a few years ago. The following paragraphs describe the author's experience with a first-generation Pilatus 6M detector installed at the X06SA beamline at the Swiss Light Source (SLS), Villigen. The detector has 6 million pixels, each covering an area of $172 \mu \mathrm{m} \times 172 \mu \mathrm{m}$. The high speed of the Pilatus (up to 10 frames per second) allows continuous, shutter-free operation with a negligible read-out time of a few milliseconds per frame. Three examples will be discussed to demonstrate the new possibilities pixel detectors offer. The results will be compared to in-house and synchrotron experiments with alternative area detectors.

The first example illustrates the capacity of pixel detectors to suppress fluorescence scattering, which is omnipresent in experiments with samples containing heavy elements. Fig. 1 shows diffraction patterns from an icosahedral $\mathrm{Al}_{64} \mathrm{Cu}_{23} \mathrm{Fe}_{13}$ quasicrystal. ${ }^{[3]}$ The two images were taken with an incident beam energy of $16 \mathrm{keV}$ under nearly the same experimental conditions: in the measurement shown in Fig. 1a photons with an energy less than $8 \mathrm{keV}$ were suppressed, while this threshold energy was increased to $10 \mathrm{keV}$ for the measurement shown in Fig. 1b. Therefore, the second experiment allowed suppression of $\mathrm{Cu}-\mathrm{K} \alpha$ and $\mathrm{Cu}-\mathrm{K} \beta$ fluorescence radiation, which is in the range from 8.03 to $8.90 \mathrm{keV}$. Note that these energies are far smaller than the primary beam energy, i.e. the fluorescence signals are not extraordinarily strong. Nevertheless, the difference between the two experiments is dramatic: while the diffuse scattering is almost completely hidden by fluorescence radiation in Fig. 1a, it is clearly visible in Fig. 1b. Suppression of fluorescence radiation was indispensable for detailed investigations of the diffuse and weak Bragg scattering from this compound. Note that the quality of measurements with energy integrative detectors, such as film, CCDs and image plates, would be limited to that shown in Fig. 1a. The concealment of diffuse scattering by a dominant fluorescence background may have been the reason why $i$ - $\mathrm{Al}_{64} \mathrm{Cu}_{23} \mathrm{Fe}_{13}$ was previously considered a rare example of a 'perfect' quasicrystal that appeared to show no diffuse scattering coming from structural disorder, and was interpreted as supporting the hypothesis that quasicrystalline structures might be stable even without a significant entropy contribution to the free energy. It is still an open question whether or not this is possible. In any case, for $i$ - $\mathrm{Al}_{64} \mathrm{Cu}_{23} \mathrm{Fe}_{13}$ our experiment contradicts this hypothesis.

The second example highlights the differences between standard in-house experiments with CCDs and image plates on the one hand and cutting edge synchrotron experiments with pixel detectors on the other hand. The compound PbTe forms a NaCltype average structure. It shows some interesting paraelectric and thermoelectric properties, which may be explained in terms of its local structure properties and corresponding entropy effects. ${ }^{[4]}$ Although $\mathrm{Pb}$ and $\mathrm{Te}$ are strong scatterers, the diffuse scattering from this system is very weak, because there is no substitutional disorder present and the average displacement amplitudes are small, only a few tenths of an Ångström $\left(\mathrm{U}_{\text {iso }}(\mathrm{Pb})=0.020 \AA^{2}\right.$ and $\mathrm{U}_{\text {iso }}(\mathrm{Te})$ $=0.014 \AA^{2}$ at room temperature $\left.{ }^{[5]}\right)$. The same crystal was measured with a CCD detector installed on a standard single crystal diffractometer (Oxford Diffraction Xcalibur diffractometer, Onyx detector, graphite monochromator, sealed tube Mo$\mathrm{K} \alpha$ radiation), with a Mar300 image plate diffractometer (rotating Mo-anode generator, $\mathrm{SiO}_{2}$ monochromator) and finally with the Pilatus 6M at SLS (wavelength 0.70846 $\AA$ ). In the latter experiment fluorescence scattering was carefully suppressed. In the hope that the diffuse scattering would become clearly visible the in-house experiments were done with extraordinarily long exposure times $(220 \mathrm{~s} /$ frame, $70 \mathrm{~h}$ in total with the CCD and 1800 s/frame, 17 days in total with the image plate). Fig. 2 shows

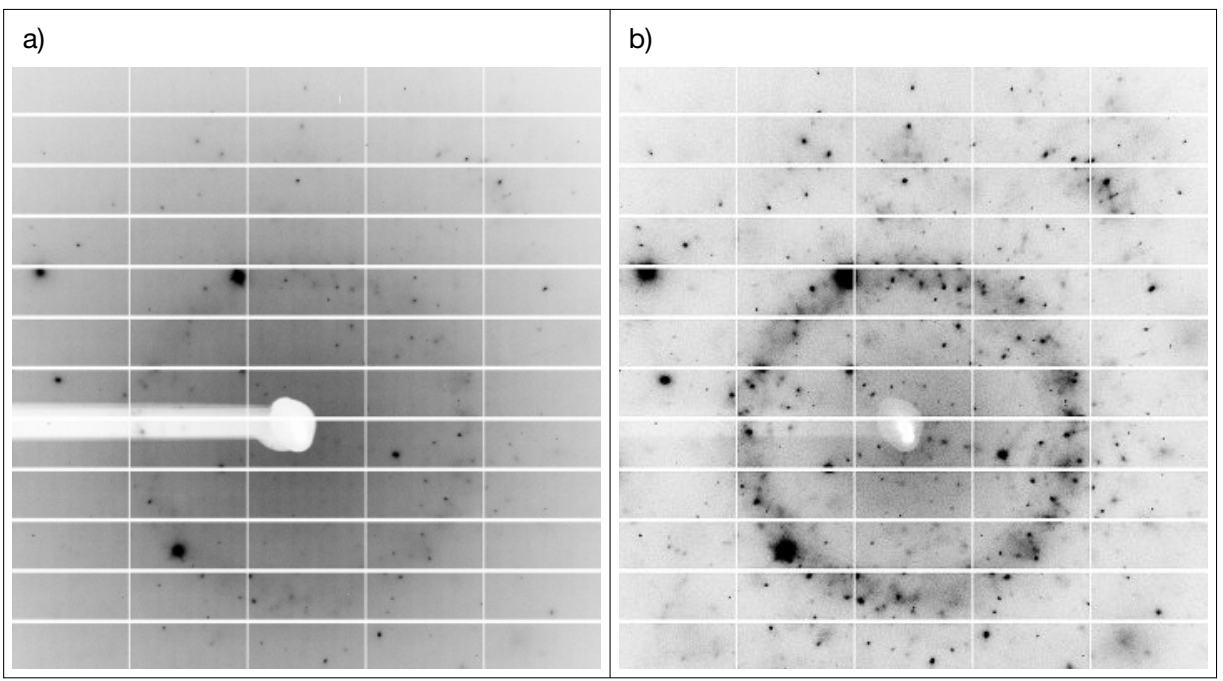

Fig. 1. Diffraction patterns of $i-\mathrm{Al}_{64} \mathrm{Cu}_{23} \mathrm{Fe}_{13}$ recorded with a Pilatus $6 \mathrm{M}$ a) without and b) with suppression of fluorescence scattering from $\mathrm{Cu}$. The white grid on the patterns is due to gaps between the detector modules. The figure is taken from ref. [3]. the diffraction patterns at room temperature. The CCD experiment did not show any diffuse scattering and the image plate experiment revealed only a small fraction of the diffuse scattering. In the synchrotron/Pilatus experiment the diffuse pattern could be measured in detail within a total experimental time of a mere six minutes (0.1 s/frame exposure time). Given the quite diverse experimental setups a direct comparison of the performance of detectors and sources is difficult. A few (i) Comprehensive measurements of very weak diffuse signals can hardly be done with in-house equipment even if the invested experimental time is far longer than that for typical Bragg experiments. (ii) The small active area of most CCDs (here 126 $\mathrm{mm}$ diameter) requires multiple measurements under different crystal orientations and different background conditions. The result is a patchwork-like pattern in the reciprocal space reconstructions that make accurate measurements of the weak diffuse signals difficult. (iii) CCD experiments do not benefit from long exposure times as much as image plate experiments. CCDs accumulate dark current proportional to the exposure time, while detector noise from image plate experiments is exclusively coming from the read-out procedure and is therefore independent of the exposure time per frame. In high dose experiments fore relatively small compared to external experimental background from air or fluorescence scattering. (iv) The combination of zero detector background, suppression of fluorescence scattering and the good reciprocal space resolution even in the vicinity of strong Bragg peaks make the Pilatus detector very well suited for measurements of weak signals at a synchrotron. conclusions may nevertheless be drawn. the detector noise on image plates is there- 


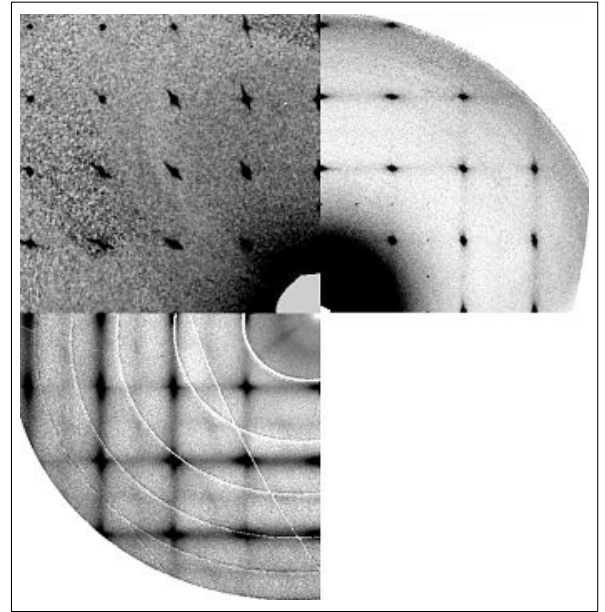

Fig. 2. Room temperature diffraction patterns of the hk0 layer from PbTe. Top left: Onyx CCD measurement, top right Mar300 image plate measurement and bottom left Pilatus $6 \mathrm{M}$ measurement. The radial streaks in the CCD experiment are due to the broad spectral range accepted by the graphite monochromator, the very weak and sharp spots close to the sphere of air scattering in the Mar300 experiment are artifacts from higher harmonics in the primary beam (' $\lambda / 2$ effect') and the white rings in the Pilatus experiment are due to the gaps between the detector modules.

The third example compares two measurements of diffuse scattering from $\mathrm{o}^{\prime}-\mathrm{Al}_{13} \mathrm{Co}_{4} \cdot{ }^{[6]}$ Experiments with the same crystal were done with the Pilatus $6 \mathrm{M}$ detector at SLS and with a Mar345 image plate at the Swiss-Norwegian Beam Lines (SNBL) at the synchrotron ESRF in Grenoble, France. From the average structures it was known that only a small fraction of the $\mathrm{Al}$ atoms is disordered. It is therefore not surprising that we completely missed the diffuse signals in our in-house experiments (not shown). However, even at SNBL we were unable to find any traces of diffuse scattering on the image plate frames (Fig. 3a). We did succeed with the Pilatus $6 \mathrm{M}$ at SLS. Within a small fraction of the time used for the image plate experiment (6 min vs. $2.5 \mathrm{~h}$ ) we could clearly measure the diffuse diffraction pattern. In addition we could identify very weak superstructure reflections that were not visible in the image plate experiments (Fig. 3b). One might argue that the superior results from the Pilatus 6M experiment might be due to the higher primary beam intensity at the X06SA beam line, which is equipped with an undulator, in contrast to SNBL, which receives its radiation from a bending magnet. Although a Pilatus 2M detector with properties similar to those of the Pilatus $6 \mathrm{M}$ was recently installed at SNBL, we have not yet compared experiments at the two beam lines systematically and a direct comparison of the primary intensities is therefore difficult. However, our experience shows that the effective flux that we observe in our experiments at SNBL is about ten to twenty times smaller than at the X06SA. In the o'- $\mathrm{Al}_{13} \mathrm{Co}_{4}$ experiments the scan speed was five times slower at $\operatorname{SNBL}\left(5 \mathrm{~s} /{ }^{\circ}\right.$ vs. $\left.1 \mathrm{~s} /{ }^{\circ}\right)$, i.e. the effective dose on the crystal was only about two to four times smaller. We therefore conclude that the differences between the Pilatus and image plate diffraction patterns cannot be explained entirely by the higher primary intensities in the Pilatus@ X06SA experiment. The better performance is more likely explained largely by the lower background in the Pilatus experiments. The experimental background level in the image plate experiment was about 20 counts per pixel. Due to fluorescence suppression and zero noise contributions from the detector, the average background in the Pilatus experiment was significantly below 1 count per pixel, if one ignores the relatively strong air scattering close to the center. Owing to the low experimental background, the average diffuse scattering signal in the Pilatus experiment is visible even though it is very weak (1-2 counts per pixel). It is not surprising that this weak diffuse scattering was hidden by the much higher experimental background in the image plate experiment.

The shape of Bragg reflections is usually unimportant because Bragg intensities are integrated quantities. In contrast the reciprocal space resolution is an important experimental factor for accurate diffuse scattering measurements. The experimental resolution function blurs the features of diffuse scattering thus reducing the disorder information obtainable from data analysis. ${ }^{[7]}$ Because of the long read-out times the image plate experiment had to be done with oscillation angles of $1 \%$ frame implying a poor resolution function. This explains why Bragg reflections are not seen
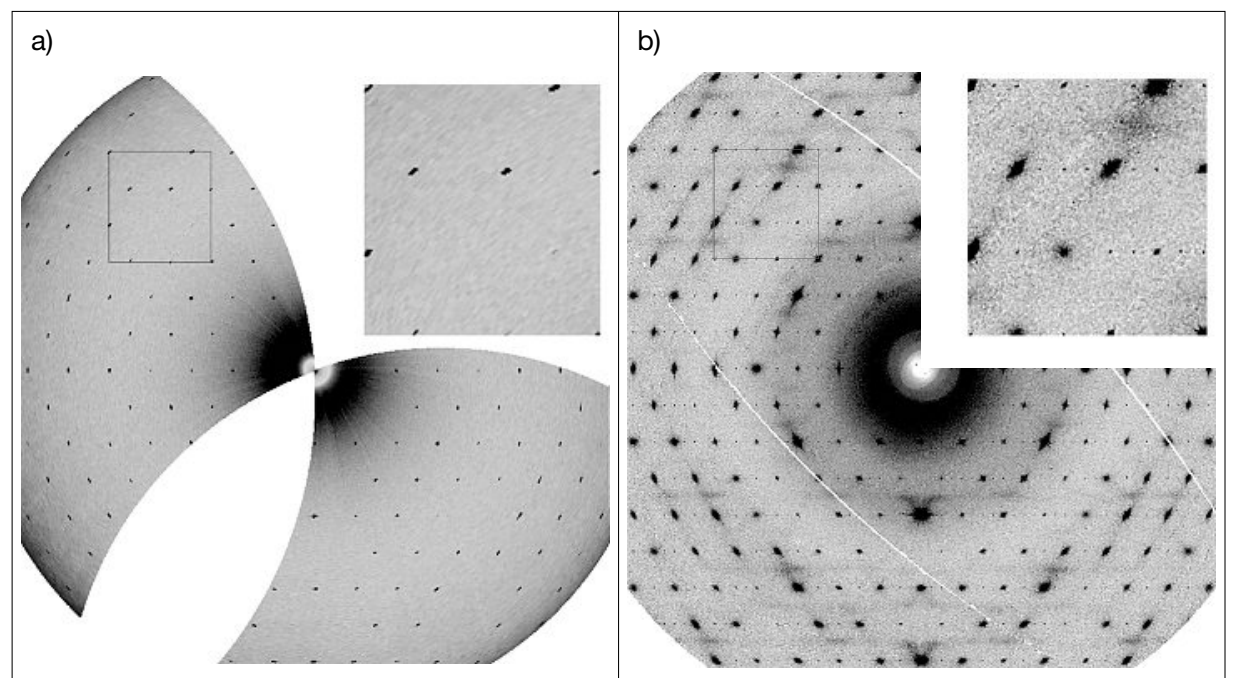

Fig. 3. Diffraction patterns from o'- $-\mathrm{Al}_{13} \mathrm{Co}_{4}$ a) with a Mar345 image plate diffractometer at SNBL@ ESRF and $b$ ) with a Pilatus $6 \mathrm{M}$ detector installed at X06SA@SLS. The top right insets show mag-

nifications of the regions indicated by the black rectangle. as sharp spots, but smeared out into small arcs (Fig. 3a). In the Pilatus experiment the frames could be recorded with a much smaller oscillation angle of $0.1 \%$ frame and without any significant overhead due to readout: 3600 frames were recorded in 6 min. A Mar345 experiment with the same experimental parameters would have taken $80 \mathrm{~h}$ just to read out the detector 3600 times. Note that most of the Bragg peaks appear to be broader in the Pilatus experiment. This is due to relatively narrow diffuse scattering beneath the peaks, which is dominating the visual impression of the diffraction pattern. The true profiles of the Bragg reflections are better seen from the weak superstructure reflections, which are free from visible diffuse scattering. Thus the Pilatus experiment not only uncovered the diffuse scattering, but it also showed a significantly improved reciprocal space resolution.

\section{Advances in Diffuse Scattering Modeling}

Before fast computers with sufficient memory became available, modeling of diffuse scattering was mainly restricted to simple one-dimensionally disordered structures, which allowed closed mathematical descriptions of disorder and corresponding diffraction intensities. ${ }^{[8]}$ However, this approach is applicable to a small class of problems only, because the mathematical expressions quickly become very complicated even in cases of one-dimensional disorder problems. ${ }^{[9]}$ An alternative approach, which was used in the past for solving disordered structures, was to compare experimental diffraction patterns to a catalogue of diffraction patterns which was generated optically with 
diffraction masks of prototypic one- or two-dimensional disorder models printed on slides. ${ }^{[10]}$ Though very useful for classification of observations and for educational purposes, this approach rarely allowed comprehensive real structure descriptions.

Parallel to the experimental advances, significant progress in data interpretation was achieved in the 1990s when computers became sufficiently fast to test model hypotheses by numerical simulations. Crystal structures were built in the computer memory using rule-based algorithms such as Monte Carlo (MC) simulations. ${ }^{[11]}$ In such simulations the building rules are expressed in terms of interaction energies between the disordered parts of the crystal structure. The model crystal is then modified in the computer memory until it reaches an energy minimum, i.e. fulfills the rules optimally. After convergence, the Fourier transform of the resulting crystal is compared to the experimental diffuse scattering to evaluate the quality of the model and the corresponding rules. The demands on the computational resources are significant: since disorder is a statistical property, the model crystals need to be very large frequently encompassing on the order of hundreds of thousands of atoms - if they are to be representative of the real crystal. Finding proper rules and optimizing the corresponding numerical parameter values was initially done by trial-and-error. With the fast improvements in computer technology, optimization of the parameter values can now be left to the computer using local and global optimization techniques. ${ }^{[11]}$ The development of proper disorder models, however, has still to be done by the scientist, since powerful algorithms analogous to direct and charge-flipping methods are not yet available for the determination of disordered structures.

A potential candidate for computersupported identification of disorder models was the Reverse Monte Carlo (RMC) method. ${ }^{[12]}$ The basic idea behind RMC is similar to MC modeling, except that the model crystal is not modified with the goal of satisfying MC construction rules, but rather with the goal of optimizing the fit to the experimental diffraction intensities. In its basic variant a priori knowledge or chemical constraints as used in MC models are not considered in the RMC procedure. Although RMC simulations quickly converge to good or even excellent agreement between observed and calculated intensities they are prone to fit experimental artifacts and to lead to unreasonable structural results. ${ }^{[13]}$ The explanation for this contradictory observation is that the number of atoms and thus the number of independent variables of an RMC model by far exceeds the number of independent measurements. Some of these problems may be overcome by adding chemical constraints to the RMC model, ${ }^{[14]}$ but the more constraints are used the more similar RMC and direct MC modeling become.

An unexpected break-through in local structure research was achieved in the 1990s. The Pair Distribution Function (PDF) technique was developed on the basis of powder diffraction, which so far was not the experimental method of choice for investigating real structure properties. Information on structural disorder is extracted directly from the Fourier transform of the powder diffraction pattern. ${ }^{[15]}$ In the meantime the powder PDF method has become very popular: the experiments are simple and fast, computing times for modeling are short, powerful software for data reduction and modeling has become available, and, last but not least, the installation of several dedicated synchrotron and neutron beam lines has rendered powder PDF studies an almost routine method, at least for relatively simple systems with small lattice constants. A shortcoming of the method is the intrinsic angular projection of the diffraction pattern to one dimension. This implies that only short interatomic vector can be resolved, since the probability of overlaps quickly increases the longer the interatomic vectors become.

The next logical step was to extend the powder PDF technique to a single crystal method. ${ }^{[7]}$ The single crystal PDF is obtained from the Fourier transform of the full single crystal diffraction pattern ('3D-PDF', analogous to a Patterson function) or from the diffuse part alone ('3D- $\triangle \mathrm{PDF}$ ' or difference Patterson function). The advantage of the $3 \mathrm{D}-\triangle \mathrm{PDF}$ over the full 3D-PDF is that it only shows the deviations from the usually well-known average structure; this simplifies modeling and computations significantly. Single crystal PDF approaches overcome most of the problems inherent to the powder PDF technique. The full three-dimensional information on the length and spatial orientation of interatomic vectors is preserved and overlap problems do not increase with increasing distances. On the other hand, high-quality three-dimensional experiments are slower and more complicated than essentially one-dimensional powder diffraction measurements.

Compared to MC the 3D- $\triangle \mathrm{PDF}$ modeling has advantages and disadvantages. On one hand, model refinements are often orders of magnitude faster. This is mainly due to the fact that PDF refinements are not hampered by speckle-type noise in the calculated, reciprocal space diffraction pattern. Such noise is unavoidable in diffraction patterns calculated from MC models. In practice full $3 \mathrm{D}-\triangle \mathrm{PDF}$ refinements may be done in minutes or hours compared to the days or weeks of CPU time typically required in $\mathrm{MC}$ refinements. On the other hand PDF methods only provide information about the pair correlations in a crystal, whereas MC models deliver representative portions of the disordered crystal. In general, MC models require fewer parameters than 3D-PDF disorder models and enable extraction of additional information. In this sense MC and PDF methods are complementary. The $3 \mathrm{D}-\triangle \mathrm{PDF}$ is very well suited for quick qualitative and quantitative explorations of disorder models, because it is very fast. If the results from a $3 \mathrm{D}-\triangle \mathrm{PDF}$ refinements are insufficient, the disorder model may be complemented by subsequent MC simulations based on the $3 \mathrm{D}-\triangle \mathrm{PDF}$ results.

The 3D- $\triangle$ PDF method was first applied to local structure investigations of quasicrystals, which are difficult to model with alternative techniques. ${ }^{[16]}$ The $3 \mathrm{D}-\Delta \mathrm{PDF}$ provides the opportunity to easily separate short and long interatomic vectors. In other words, the determination of the real atomic structure of the disordered building units ('clusters') of quasicrystals may be separated from modeling the long-range spatial distribution of such clusters. While the clusters cover small volumes in threedimensional space and are therefore well suited for 3D- $\triangle \mathrm{PDF}$ studies, their longrange distribution is more easily described in five- or six-dimensional space.

\section{An Example: Disorder in $\operatorname{La}_{0.70}\left(\mathrm{Al}_{0.14} \mathrm{I}_{0.86}\right)$}

The structure of the non-stoichiometric compound $\mathrm{La}_{0.70}\left(\mathrm{Al}_{0.14} \mathrm{I}_{0.86}\right)$ is a typical example of a disordered crystal where the average structure alone allows almost no conclusions about the chemical building blocks of the material. ${ }^{[17]}$ The compound forms a NaCl-type structure in which the cation sites are partially occupied by La and the anion sites have mixed occupation by $\mathrm{Al}$ and I. Minor displacements from the ideal $\mathrm{NaCl}$ Wyckoff positions indicate the presence of local relaxations. It is well known that such metal-rich rare-earth halides frequently form so-called $\mathrm{M}_{6} \mathrm{X}_{12} \mathrm{Z}$ clusters. ${ }^{[18]}$ If this would also be true in the present case a central $\mathrm{Al}$ atom $(=\mathrm{Z})$ would be octahedrally coordinated by six La atoms and by $6+6$ iodine atoms, which are separated from the $\mathrm{Al}$ positions by $<0.5$ $0.50>$ and $<100>$ vectors corresponding to the so-called 'inner' and 'outer' ligands, respectively. The number of ligands per cluster is reduced if $\mathrm{AlLa}_{6}$ octahedrons share edges or corners (Fig. 4). Due to serious disorder it is not possible to conclude from the average structure whether or not AlLa 6 octahedrons exist in $\mathrm{La}_{0.70}\left(\mathrm{Al}_{0.14} \mathrm{I}_{0.86}\right)$, how the clusters - if they are present - are distributed in the crystal, and how the 
atomic displacements correlate with the local structure. In other words, the chemical information of interest is almost completely obscured by the disorder in the average structure. To obtain the real structure information diffuse scattering experiments were performed with a Mar345 image plate diffractometer $(\mathrm{Ag}-\mathrm{K} \alpha$ radiation, graphite monochromator). Note that the very strong diffuse intensities allowed in-house measurements of sufficient quality. The interpretation of the diffuse scattering was done with MC modelling using a home-written ad-hoc program. Details of the MC models have been described in ref. [17b]. Fig. 5 shows that the agreement between observed and calculated diffuse intensities resulting from the refinement is very good. The final MC model delivered a wealth of information beyond the average $\mathrm{NaCl}$-type structure. It has not only confirmed that $\mathrm{Al}$ is fully coordinated by La, i.e. that $\mathrm{AlLa}_{6}$ octahedrons are present, but has also furnished detailed information about relaxations within the clusters and about the coordination and distribution of the clusters. It was found that the $\mathrm{La}$ atoms and the outer ligands move towards the center of a cluster, while the inner ligands move away from the endrohedral $\mathrm{Al}$ atom along radial directions. Corner- and edge sharing cluster arrangements are rare, while clusters pairs, which are not condensed, but have a void cation site in between them are favored. A detailed description of the complex ordering in $\mathrm{La}_{0.70}\left(\mathrm{Al}_{0.14} \mathrm{I}_{0.86}\right)$ is reported in ref. [17b].

\section{Summary and Outlook}

In the last twenty years progress in synchrotron, detector and computer technology has revolutionized diffuse scattering research. With electronic area detectors such as CCDs and image plates, sufficient synchrotron beam time and powerful computers quantitative investigations of local structures have become available to a broader community. However, diffuse scattering studies were still tedious and far from routine. Obtaining high quality diffuse scattering data was time consuming or not possible at all, if the diffuse signals were weak. Interpretation of the data was impractical if disorder was complex and computing times for modeling long. The most recent developments such as third generation synchrotrons, pixel detectors, new modeling algorithms and clusters of ultra fast computers promise to overcome the last bottlenecks impeding more routine investigations of diffuse scattering. Pixel detectors are already installed at a number of synchrotron beam lines and will likely become widely available in in-house laboratories as well. Compared

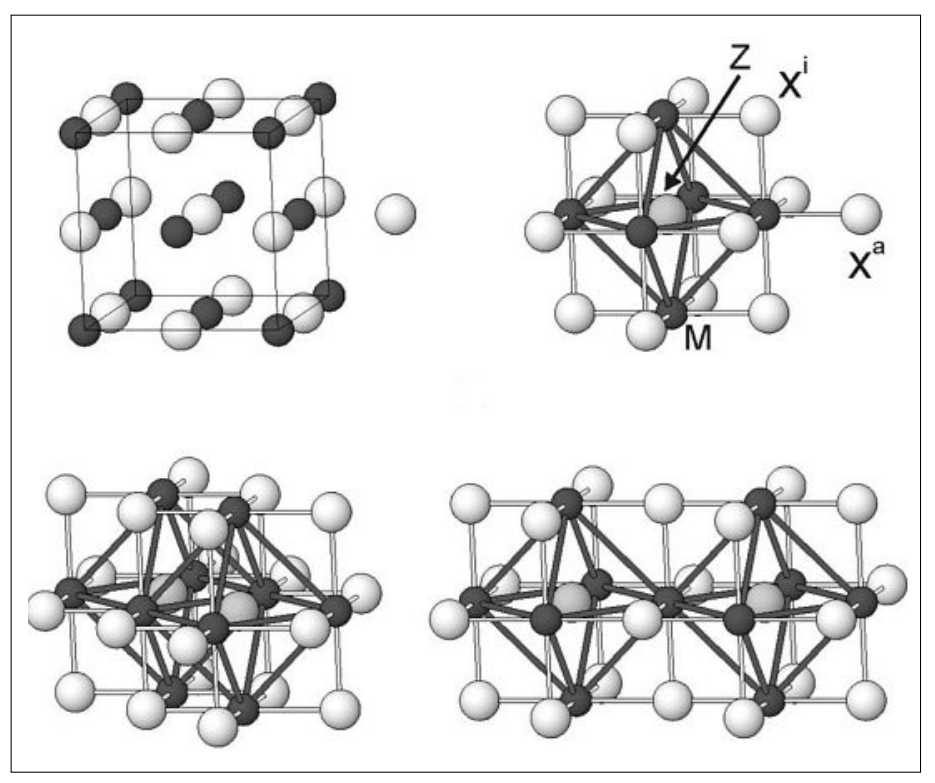

Fig. 4. Top: comparison of an isolated $\mathrm{M}_{6} \mathrm{X}_{12} \mathrm{Z}$ cluster (right) with the average $\mathrm{NaCl}$-type structure (left). Bottom: edge and corner-sharing condensed clusters. The figure is taken from ref. [17b].

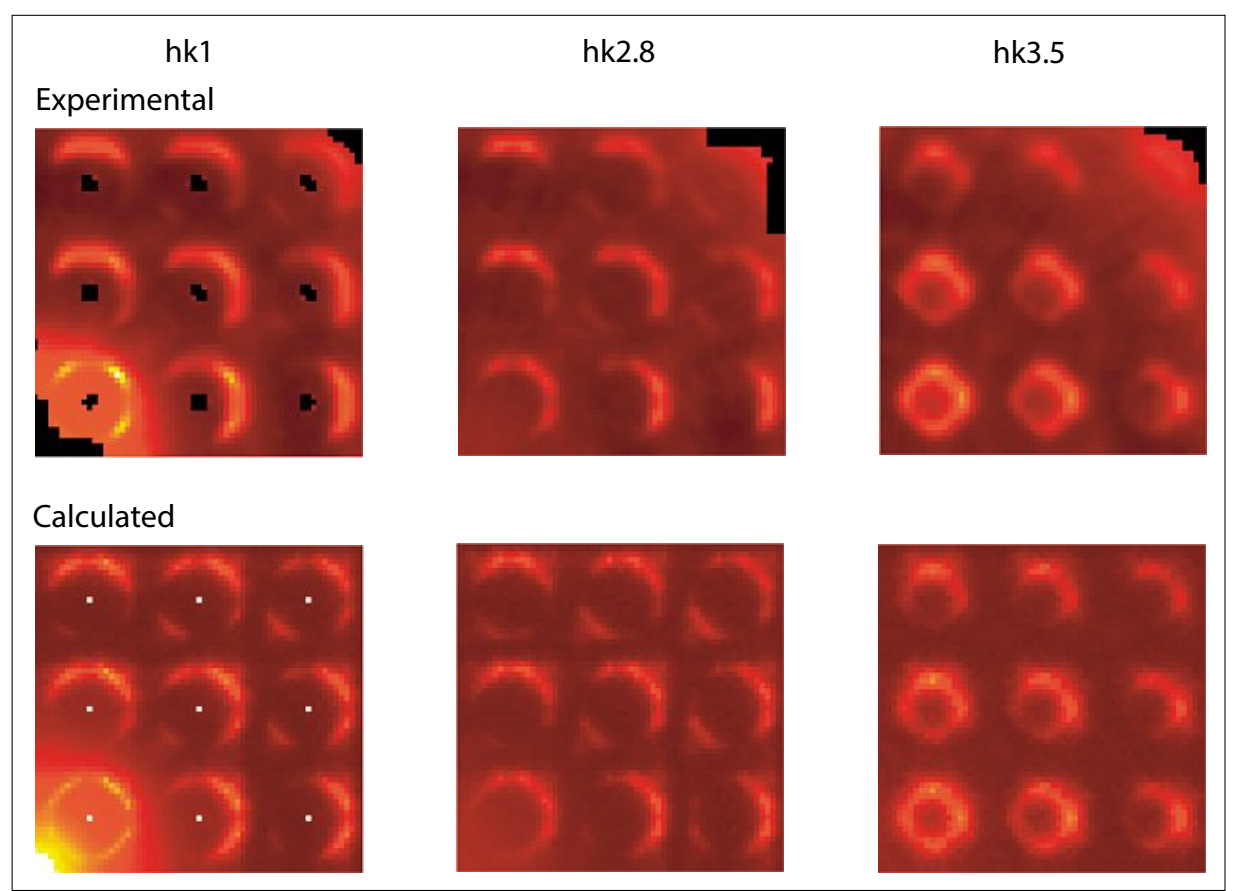

Fig. 5. Sections from observed and calculated diffraction patterns of $\mathrm{La}_{0.70}\left(\mathrm{Al}_{0.14} \mathrm{I}_{0.86}\right)$. The diffuse arcs and rings are sections through strongly modulated hollow spheres around Bragg reflections with indices $h, k, I=$ all odd. The figure is taken from ref. [17b].

to the first generation Pilatus 6M used in our experiments, the newest generation pixel detectors have smaller pixel sizes for better reciprocal space resolution. They cover larger dynamic ranges for simultaneous measurements of Bragg and diffuse data; attenuating the beam to $10-20 \%$ of the available intensity to avoid saturations as in our previous experiments with the Pilatus 6M will no longer be necessary. Measurements of up to 500 frames per second will further reduce experimental times. It is expected that the newest generation of pixel detectors will enable us to take advantage of the full power of the beam and to measure complete and high-quality single crystal data sets within less than a minute. Time-resolved measurements of slow dynamic processes and experiments under critical non-ambient conditions will become much easier.

MC modeling software currently being developed includes the programs ZMC, [19] which supports modeling of organic structures using the z-matrix description, and ZODS, ${ }^{[20]}$ which takes advantage of big cluster and supercomputers to parallelize the notoriously slow MC simulations and to make them significantly faster in terms of wall-clock time. ${ }^{[21]}$ Furthermore, the $3 \mathrm{D}-\triangle \mathrm{PDF}$ modeling and least-squares refinement program Yell[22] is now in its testing phase. It was already successfully applied to a number of real world problems ${ }^{[23]}$ and will be made available to the community soon. 
The potential for more routine investigations of local structure has been clearly demonstrated by the remarkable success of the powder PDF method. It is becoming increasingly clear that in the near future single crystal diffuse scattering studies will go far beyond the current successes attained with powder data. Given recent achievements with the tools that are currently being developed single crystal diffuse scattering research has the potential to become a more accessible technique, which will surmount the barriers imposed by averaging over all unit cells of a crystal. It may extend the realm of structure research to disordered crystal structures of almost any chemical and biological molecular compound, multinary phase and other material which owes some or all of its important and useful properties to structural disorder.

\section{Acknowledgement}

The author thanks the staff of the SwissNorwegian Beamlines at ESRF, Grenoble and of the X06SA beamline at SLS, Villigen for their friendly support and for providing generous access to the instruments.

Received: November 27, 2013

[1] M. A. Estermann, W. Steurer, Phase Transitions 1998, 67,165
[2] C. Broennimann, E F. Eikenberry, B. Henrich, R. Horisberger, G. Huelsen, E. Pohl, B. Schmitt, C. Schulze-Briese, M. Suzuki, T. Tomizaki, H. Toyokawa, A. Wagner, J. Synchrotron Rad. 2005, 13, 120.

[3] T. Weber, S. Deloudi, M. Kobas, Y. Yokoyama, A. Inoue, W. Steurer, J. Appl. Cryst. 2008, 41, 669.

[4] a) E. S. Bozin, C. D. Malliakas, P. Souvatzis, Th. Proffen, N. A. Spaldin, M. G. Kanatzidis, S. J. L. Billinge, Science 2010, 330, 1660; b) K. M. Ø. Jensen, E. S. Bozin, C. D. Malliakas, M. B. Stone, M. D. Lumsden, M. G. Kanatzidis, S. M. Shapiro, S. J. L. Billinge, Phys. Rev. B 2012, 86, 085313; c) Y. Zhang, X. Ke, P. R. C. Kent, J. Yang, C. Chen, Phys. Rev. Lett. 2011 107, 175503; d) O. Delaire, J. Ma, K. Marty, A. F. May, M. A. McGuire, M-H. Du, D. J. Singh, A. Podlesnyak, G. Ehlers, M. D. Lumsden, B C. Sales, Nature Materials 2011, 10, 614

[5] Y. Noda, K. Masumoto, S. Ohba, Y. Saito, K. Toriumi, Y. Iwata, I. Shibuya, Acta Cryst. C 1987, C43, 1443.

[6] F. Fleischer, T. Weber, D. Y. Jung, W. Steurer, $J$. Alloys Compd. 2010, 500, 153.

[7] T. Weber, A. Simonov, Z. Kristallogr. 2012, 227, 238.

[8] a) H. Jagodzinski, Acta Cryst. 1949, 2, 201; b) H. Jagodzinski, Acta Cryst. 1949, 2, 208; H. Jagodzinski, Acta Cryst. 1949, 2, 298.

[9] H. B. Bürgi, M. Hostettler, H. Birkedal, D. Schwarzenbach, Z. Kristallogr. 2005, 220, 1066.

[10] T. R. Welberry, C. A. Taylor, H. J. Milledge, J. Appl. Cryst. 1972, 5, 133.

[11] a) T. R. Welberry, T. Proffen, M. Bown, Acta Cryst. A 1998, 54, 661; b) T. Weber, H. B. Bürgi, Acta Cryst A 2002, 58, 526.
[12] a) R. L. McGreevy, J. Phys.-Condens Mat. 2001, 13, R877; b) T. R. Welberry, T. Proffen, J. Appl. Cryst. 1998, 31, 309.

[13] T. Weber, Z. Kristallogr. 2005, 220, 1099.

[14] J. Pikunic, C. Clinard, N. Cohaut, K. E. Gubbins, J.-M. Guet, R. J.-M. Pellenq, I. Rannou, J.-N. Rouzaud, Langmuir 2003, 19, 8565.

[15] S. J. L. Billinge, T. Egami, Phys. Rev. B 1993, 47, 14386.

[16] a) M. Kobas, T. Weber, W. Steurer, Phys. Rev. B 2005, 71, 224205; b) M. Kobas, T. Weber, W. Steurer, Phys. Rev. B 2005, 71, 224206; c) T. Weber, M. Kobas, W. Steurer, Ferroelectrics 2004, 305, 213; d) P. Schaub, T. Weber, W. Steurer, J. Appl. Cryst. 2011, 44, 134.

[17] a) O. Oeckler, T. Weber, L. Kienle, H. J. Mattausch, A. Simon, Angew. Chem. Int. Ed. 2005, 44, 3917; b) T. Weber, A. Simon, H. J. Mattausch, L. Kienle, O. Oeckler, Acta Cryst. A 2008, 64, 641 .

[18] A. Simon, Angew. Chem. Int. Ed. 1988, 27, 160.

[19] D. J. Goossens, A. P. Heerdegen, E. J. Chan, T. R. Welberry, Metall. Matter. Trans. A 2011, 42, 23.

[20] M. Chodkiewicz, T. Weber, L. Ahrenberg, H. B. Bürgi, University of Zurich.

[21] T. M. Michels-Clark, V. E. Lynch, C. M. Hoffmann, J. Hauser, T. Weber, R. Harrison, H. B. Bürgi, J. Appl. Cryst. 2013, 46, 1616.

[22] A. Simonov, T. Weber, W. Steurer, ETH Zurich.

[23] a) J. Dshemuchadse, S. Bigler, A. Simonov, T. Weber, W. Steurer, Acta Cryst. B 2013, 69, 238; b) T. Weber, A. Simonov, in preparation; c) P. Urban, A. Simonov, T. Weber, O. Oeckler, in preparation. 